\title{
Pediatric Gastric Bypass: Is it Time to Rethink? Lessons Learned from a Single Institution Experience
}

\author{
Keywords: Gastric bypass; Pediatric patients; Adolescent; \\ Outcomes

\begin{abstract}
Pediatric obesity is a growing problem in United States. Ideally, a non-operative option is preferred but currently there is no medica treatment that guarantees an enduring weight loss. Laparoscopic adjustable gastric banding is popular in some centers due to its safety profiles. Due to the limited experience, gastric bypass, which has shown excellent outcomes in adult population, is less popular in the pediatric population.
\end{abstract}

Aims: To evaluate our initial experience with gastric bypass in adolescent population.

Patients and methods: Between J anuary 2008 and July 2010, 19 adolescents with morbid obesity underwent laparoscopic Roux gastric bypass with a Roux limb of $140 \mathrm{~cm}$.

Results: Mean age 18 years (range: 16-20 years), Male: female ratio $=1: 18$, Mean weight $=305 \mathrm{lb}$ (range $=230-384)$, Mean $B M l=50$ (range: $42-67$ ). Mean Percentage excess weight loss was $88 \%$ and $74 \%$ at 1 and 2 years respectively. No patient was lost to follow- up. There wasa nearcomplete resolution of a lmost all associa ted co-morbidities.

Four complications occurred during the study period (1intema hemia, I surgic al site infection, 1 hypoglycemic episode and 1 stomal stenosis). Of these, only one patient required reoperation.

Conclusion: Although our initial experience is limited by the smal sample size, we believe that our results are comparable to those reported in adult population. We believe that gastric bypassis a viable option altemative in the adolescent population.

\section{Introduction}

Worldwide, the incidence of obesity is growing. In the United States, the prevalence of pediatric obesity has increased from $4 \%$ in 1971 to $15 \%$ in 2007 [1].

Childhood obesity predisposes to hyperlipidemia, hypertension, insulin resistance and type 2-diabetes mellitus (T2DM), degenerative joint disease as well as liver, renal and menstrual dysfunction. These complications have significant impact on quality of life and may reduce life expectancy. Childhood obesity represents a precursor of adult obesity, as $50-70 \%$ of these adolescents will become obese adults [2-4]. Unless this trend is halted, the health and economic implications on global economy will be severe.

The optimal treatment for adolescent obesity is yet to be determined. Data are lacking but still available to draw general conclusions. Available medical treatments are unpredictable.

The two most commonly used and well-studied procedures for adolescents are the adjustable gastric band and the Roux-en-Y gastric

\section{Journal of Obesity and Bariatrics}

\author{
Emmanuel A. Agaba*, Harvey Rainsville and \\ Prathiba Vemulapalli
}

Department of Bariatric Surgery, Montefiore Medical Center at Albert Einstein College of Medicine, Bronx, New York, USA

\section{*Address for Correspondence}

Dr Emmanuel A. Agaba, MD, Department of Bariatric Surgery, Montefiore Medical Center at Albert Einstein College of Medicine, Bronx, New York 10467, USA, Tel: 718-9206755; E-mail: eagaba@gmail.com

Copyright: (c) 2015 Agaba EA, et al. This is an open access article distributed under the Creative Commons Attribution License, which permits unrestricted use, distribution, and reproduction in any medium, provided the original work is properly cited.

Submission: 29 December 2014

Accepted: 13 February 2015

Published: 18 February 2015

bypass. Of these, gastric bypass is the only approved surgical option for pediatric population in the USA. While both gastric bypass and banding are effective in treating the medical consequences of obesity in adolescents, gastric bypass surgery has been shown to be the most effective for optimal weight loss. Because the long-term effects of gastric bypass in pediatric population are largely unknown, some surgeons prefer laparoscopic adjustable gastric band (LAGB) as the primary option in adolescent patients [5]. LAGB is not effective in all patients and some patients will inevitably require a second procedure. More recently, O'Brien and colleagues from Australia reported a 33\% reoperation rate for patients aged 14-18 years who underwent LAGB in a prospective randomized trial [6].

Unlike the European and Australian experience, LABG outcome data in US are variable [6-8]. Yet these results were extrapolated to the adolescent population. For this reason a surgical option that guarantees an enduring weight loss is desired.

\section{Aims}

This study was designed to evaluate our initial experience with 19 morbidly obese adolescent patients who underwent laparoscopic gastric bypass and to compare our result with published results in adult population.

\section{Patients and Methods}

This is a retrospective review of medical records of all patients 20 years or under who underwent laparoscopic Roux Y gastric bypass (RYGB) between January 2008 and July 2010 in a high volume Bariatric Center of Excellence in Bronx, New York. In accordance with the Endo Panel Obesity guidelines, we defined morbid obesity in adolescents as Body mass Index (BMI) $>95$ percentile adjusted for age, sex and ethnic group $[9,10]$ (Tables $1 \mathrm{~A}$ and $1 \mathrm{~B})$.

We defined hypertension as consistent with Systolic blood pressure (SBp) and/ or Diastolic Blood pressure (DSp) $>95$ percentile for adjusted for age, gender and height measured at 3 separate occasions [11-13]. We defined diabetes as elevated fasting blood sugar $>150 \mathrm{mg} / \mathrm{d}$ (samples were analyzed in fresh plasma). Sleep obstructive apnea was defined as respiratory index of at least 10 hypopneic and/ or apneic episodes per hour of sleep. Gastro esophageal reflux disease 
Citation: Agaba EA, Rainsville H, Vemulapalli P. Pediatric Gastric Bypass: Is it Time to Rethink? Lessons Learned from a Single Institution Experience.. J Obes Bariatrics. 2015;2(1): 5.

(GERD) was defined as "heart burn" that was relieved by Proton Pump Inhibitor or $\mathrm{H}_{2}$. Blockers and seen on upper Endoscopy or upper gastrointestinal series. Degenerative joint disease (DJD) was defined as the presence of pain in weight bearing joint.

Resolution of hypertension was defined as SBp $<135 \mathrm{mmHg}$ or $\mathrm{DBp}<85 \mathrm{mmHg}$ and not on any antihypertensive medications, while resolution of diabetes was defined as fasting blood sugar $<120 \mathrm{mg} / \mathrm{dl}$ and in the absence of hypoglycemic drugs (samples were analyzed in fresh plasma)

All patients were initially seen by a multidisciplinary team (consisting of bariatric surgeons, pediatricians, pediatric endocrinologist, pulmonologist, pediatric psychologist, and Bariatric Nutritionist). All patients with symptoms of obstructive sleep apnea or excessive snoring underwent overnight polysomongram.

All patients were obese for at least 5 years and have documented evidence of failed attempt at medically supervised weight loss program for 6 months. In preparation for surgery, all patients underwent upper Endoscopy with biopsy and had satisfactory psychological evaluation. All patients underwent appropriate nutritional evaluation, including micronutrients measurements (Iron studies, Vitamin B12, 25-Hydroxy Vitamin D, Folic acid, Homocysteine, Methyl malonic acid, Albumin, Parathormone, Calcium, Copper, Zinc, Selenium, and Thiamine) before their procedure and subsequently at 3 months, 6 months and annually thereafter (Table 1C).

One patient with end stage renal failure underwent preoperative echocardiogram.

All patients were evaluated by an attending anesthesiologist before surgery. At the time of operation, all patients received a third generation cephalosporin as well as unfractionated Heparin at induction of anesthesia. Subcutaneous injection of unfractionated Heparin was continued twice daily postoperatively throughout the hospital course. Additionally, sequential compression boots were also used intraoperatively and postoperatively until ambulatory.

In performing the operation, a standard 5 port RYGB was

Table 1A: Guideline for definition of morbid obesity in adolescent.

$\mathrm{BMI} \geq 40 \mathrm{~kg} / \mathrm{m} 2$ with one or more severe obesity related co morbidities (e.g., type 2 diabetes mellitus, severe steatohepatitis obstructive slepp apnea, or pseudo tumor cerebri)

$\mathrm{BMI} \geq \mathrm{of} 50 \pm$ with minor co morbidities (hypertension, dyslipidemia,mild steatohepatitis, significant impairment in quality of life, or arthropathy)

Table 1B: Inclusion criteria.

$\mathrm{BMI} \geq 40 \mathrm{Kg} / \mathrm{M}^{2}$ with major co-morbidities

$\mathrm{BMI} \geq 50 \mathrm{~kg} / \mathrm{M}^{2} \pm$ with minor co-morbidities

Age 16-20

Tanner 4 stage of development ${ }^{14}$

Table 1C: Exclusion criteria

Major psychiatric illness that preclude willingness to follow protocol requirements.

Unwillingness to adhere to strict protocol requirements

Pregnancy, breast-feeding, or plan to become pregnant within 2 years of surgery

Substance abuse within the preceding year performed with a Roux limb of approximately $140 \mathrm{~cm}$ (range: 120$180)$.

A standard $30-50 \mathrm{ml}$ gastric pouch was created over a 32 French gauge Maloney bougie. A $2.5 \mathrm{~cm}$ side-to-side gastrojejunal anastomosis was created using linear stapler. The gastrotomy and enterotomy were closed over the bougie using continuous ethibond ${ }^{\mathbb{Q}}$ 0 sutures. In the early part of the study, the Roux limb was tunneled through the retrocolic space to reach the pouch $(\mathrm{N}=13)$, but this was changed in the later part of the study following the development of internal hernia in one patient.

The Mean operative time was 2 hours (range: 1.45-3 hours). Estimated blood loss was $30 \mathrm{ml}$ (range: 25-60 ml).

All patients were mobilized within 6hours of operation. One patient had an upper gastrointestinal series to rule out anastomotic dehiscence. All patients were commenced on clear liquid diet on postoperative day 1. All patients were discharged from hospital on postoperative day 2 when they were tolerating 3-4 oz of clear liquid diet/hour. All patients were discharged home with chewable multivitamins, Calcium, Proton pump inhibitor and Ferrous sulfate elixir. Vitamin D was added to the regimen during the first office visit. All patients were followed up closely in the clinic a week later, then at $1,3,6,12$ monthly and yearly afterward. During each visit, each patient's BMI, percent excess weight loss, ability to tolerate regular diet, resolution of co-morbidities, drug requirement and compliance were determined. At the third postpone months, blood was drawn to assess serum nutritional, biochemical, parathyroid hormone and hematological parameters.

\section{Results}

Nineteen consecutive patients ( 18 females and 1 male) underwent laparoscopic Roux Y gastric bypass (LRYGB). Mean BMI $=50$ (range: 42-67) and Mean weight of $305 \mathrm{lb}$ (range $=230-384$ ), of the 19 patients who underwent RYGB, 4 patients had hypertension (21\%), 2 patients had diabetes (11\%). One patient with diabetes also had hypertension and obstructive sleep apnea (OSA). Overall, OSA occurred in 4 patients (21\%). DJD and GERD occurred in 3 and 1 patients respectively. Hyperlipidemia occurred 3 patients (16\%).

Among hypertensive's, disease resolution occurred in $75 \%$ of the patients (Table 2). One patient with hypertension also had end stage renal failure and was the only patients whose hypertension did not resolve following her surgery. She was however taking fewer antihypertensive medications following her surgery.

Diabetes mellitus resolved in all patients, as did GERD, DJD, hyperlipidemia and OSA.

With a follow up period of 1-2 years (all 19 patients were seen), we reported percentage excess weight loss of 88 and 74 at one and two years respectively (Table 3 ).

Four patients had postoperative course complicated by internal hernia (1), surgical site infection (1), stomal stenosis (1) and hypoglycemic episode (1). No postoperative death was recorded in our series. Of the four, one patient with internal hernia underwent diagnostic laparoscopy, reduction of hernia and closure of Petersen's defect 14 months after the index procedure (after total weight loss 
Citation: Agaba EA, Rainsville H, Vemulapalli P. Pediatric Gastric Bypass: Is it Time to Rethink? Lessons Learned from a Single Institution Experience.. J Obes Bariatrics. 2015;2(1): 5.

ISSN: 2377-9284

Table 2: Disease Resolution in adolescent compared with published adult series.

\begin{tabular}{|c|c|c|c|c|c|c|c|c|}
\hline Study & No of pts & DM \% & GERD\% & H'tension\% & Sleep apnea\% & Depression \% & H'lipid\% & DJD\% \\
\hline This study & 19 & 100 & 100 & 75 & 100 & NA & 100 & 100 \\
\hline $\begin{array}{l}\text { Ocon-Breton }{ }^{15} \\
(2010)\end{array}$ & 46 & 94 & NA & 85 & NA & NA & 96 & NA \\
\hline Bowne ${ }^{16}(2006)$ & 46 & 100 & NA & 63 & 88 & NA & 43 & 29 \\
\hline $\begin{array}{l}\text { Puzziferri17 } \\
(2006)\end{array}$ & 59 & 100 & 100 & 83 & 100 & 76 & 88 & 80 \\
\hline Eid ${ }^{19}(2005)$ & 24 & 100 & 100) & 77 & NA & 100 & 92 & NA \\
\hline Schauer $^{20}(2000)$ & 275 & 82 & 72 & 70 & 74 & 8 & 63 & 41 \\
\hline
\end{tabular}

DM: Diabetes Mellitus; H'Tension: Hypertension; H'lipid: Hyperlipidemia; GERD: Gastroesophageal Reflux Disease; DJD: Degenerative Joint Disease

Table 3: Percentage excess weight loss (\%EWL) in adolescent and adult populations

\begin{tabular}{|l|c|c|c|}
\hline \% EWL & 1year & 2years & 74 \\
\hline This study & 88 & 73 & NA \\
\hline Pories $^{21}$ (1992) & 71 & NA & 62 \\
\hline O'Rourke $^{22}$ (2006) & 54 & 75 & NA \\
\hline Puzziferri $^{\mathbf{5}}$ (2008) & 70 & 72 & NA \\
\hline Agaba $^{23}$ (2008) & 74 & NA \\
\hline
\end{tabular}

in excess of $70 \mathrm{lb}$ ). Another patient developed stomal stenosis at 10 months. This patient underwent upper gastrointestinal endoscopy with balloon dilatation. One patient with superficial surgical site infection was successfully treated with antibiotic.

No 30-day readmission was reported in this series. Thereafter, we had three readmission rates during the study period. Two of the 3-readmissions were procedural related.

\section{Discussion}

Due to the potential risks of surgical weight loss surgery, absence of evidence based reports and long-term outcome data in adolescent patients, there is reluctance among pediatricians, bariatric surgeons and parents in recommending gastric bypass surgery in this group. Yet the best available alternatives such as family based behavioral modifications to support changes in diets and physical activity, endorsed by the American Academy of Pediatrics have poor success rates for adolescents with morbid obesity [6].

All studies that have reported outcome data following weight loss surgery in adolescent have shown a clinically important and durable decrease in weight and BMI with minimal risk.

While some agree that LAGB is the preferred option for adolescent because of its safety and reversibility records, others believe RYGB is preferred $[6,14,15]$. Among patients who underwent LAGB, there is a significant risk of reoperations. In a recent prospective randomized study conducted by Balsiger and colleagues [15], the reoperation rate was $33 \%$. A further $18 \%$ and $14 \%$ required band replacement or removal respectively. In a separate study, O'Brien et al. reported a similar reoperation rate [6]. Other workers reported major late complications such as pouch dilation, erosion, migration or other device related problems $[16,17]$.
RYGB offers a suitable alternative and has a more predictable excess weight loss. Although earlier report of gastric bypass/ gastroplasty by Anderson et al. in 1980 produced a modest weight loss that was not sustainable at 5 years [18]. Since then Rand and others have reported percentage excess weight loss of $53-80 \%$ at 2-6 years follow up [19-22]. In our series, we achieved a percentage excess weight loss of $88 \%$ and $74 \%$ at 1 and 2 years respectively. Our result is supported by a similar outcome reported by Stanford and colleagues [15]. In their study, Stanford reported \%EWL of $87 \%$ at 20 months [15]. In a separate report in the adult population, Agaba and associates reported \%EWL of $74 \%$ and $72 \%$ at 1 and 2 years respectively [23]. These data suggest that the long-term efficacy and safety profiles among adolescents' mirrors the outcomes in larger series in adult population [24,25].

Searching for indicators of direct clinical impact of RYGB in adolescent population is lacking. Resolutions of co-morbidities have been used as surrogate marker. In our series, we had $100 \%$ resolution of diabetic $(n=2)$, hyperlipidemia $(n=3)$, degenerative joint. Disease $(\mathrm{n}=3)$, Obstructive sleep apnea $(\mathrm{n}=4)$ and GERD $(\mathrm{n}=1)$. We achieved a $75 \%$ resolution of hypertension in our series. Although one patient remained hypertensive, there was an improvement in her condition as the number of her antihypertensive medication was reduced from three to two (patient is currently awaiting renal transplantation). These findings support other previous studies that have shown that weight loss of 5-10\% may be beneficial in decreasing cardiovascular risk and increase life expectancy among morbidly obese patients $[26,27]$.

There is a growing concern of psychosocial, sexual and skeletal immaturity among adolescent when offered RYGB at this stage of their development. In Rand's study this issue was addressed and the evidence was lacking [19]. In Rand's series, the focus of the paper was psychosocial changes and compliance with nutritional supplement 
Citation: Agaba EA, Rainsville H, Vemulapalli P. Pediatric Gastric Bypass: Is it Time to Rethink? Lessons Learned from a Single Institution Experience.. J Obes Bariatrics. 2015;2(1): 5.

after a successful weight loss surgery [19]. Most of their patients agreed that if they had to do it over again, they would. Two patients who did not achieve a satisfactory weight loss were displeased with their outcomes and would not do it over again. Most of their patients were satisfied with their improved social standing and self esteem. Their worst outcome was "nothing" followed by redundant skin and scarring. All the patients were either in full time education or are gainfully employed. Some patients were married with children. The authors concluded that although the effect of psychosocial benefit was difficult to measure, it was an inevitable gain to the patients [19].

Because RYGB is associated with metabolic and nutritional deficiencies, sexual and skeletal maturity should be attained before undertaking RYGB. In our series, the mean age was 18. Based on the Center for Disease Control growth chart, we believe that at this age an average male would have completed $98.4 \%$ of his growth to age 20 and the corresponding percentage for female is $99.6 \%$. We, like others have found no evidence of stunted growth, metabolic or hepatic dysfunction in following RYGB.

To our knowledge, there has been no evidence of negative reports surrounding the use of RYGB in adolescent. Although preoperative complications among the adolescent population parallel those in adults, there is a growing wealth of evidence that suggest it may be safer in adolescents. While the reason for this is unclear, it may reflect a better state of health among adolescents undergoing RYGB. We reported 4 complications (21\%). Our result was similar to those of Nguyen and associates who reported a complication rate of $16 \%$ among 79 adult patients [26]. Like Tsai et al. series we had no preoperative mortality and had similar length of stay that was comparable with adults $[23,28]$. We believe that this relatively low mortality rate reflects a better patient selection as well as improvement in advanced surgical skills.

One patient suffered severe postprandial hypoglycemic episode requiring treatment. Unlike in adult population where this complication is well documented [29,30], it is rare in pediatric population. To our knowledge, this is the first documented case in the English language literature. While the exact cause of this remains largely unknown, but it is widely believed to be due to pancreatic islet cell hyperplasia, elevated incretion secretion and inappropriate postprandial hyperinsulinemia [30].

Non-compliant with postoperative nutritional supplements, exercise and preoperative drug regimen raises serious concerns in the follow up of adolescent patients. Rand et al. in their study observed that only $13 \%$ of their patients were compliant with their nutritional supplements at 6 years [19]. In our experience, compliance was 95\% at 2 years. It is likely that as the patients achieved the desired weight loss, compliance will decrease with time as shown by Rand's data. The reason for the low compliance in Rand's series is hard to explain. One reason for our high compliance rate was partly due to the continuing patient education we offered during each visit as well as strong family support.

Readmission after RYGB is a major concern. In our series, we had 3patients that were readmitted during the study period. Two of the three readmission occurred during the first year and supports Dingmond's observation that readmission during the first year is procedure related [31]. In their series of 24,678 patients who underwent RYGB, Dingmond et al. observed a readmission rate of $20 \%$ at first year, $18 \%$ in the second year and $15 \%$ in the $3^{\text {rd }}$ year [31]. Readmission during the first year is procedure related. In our series all the readmissions were procedure related thus confirming Dingmond observation. Readmission during the $3^{\text {rd }}$ year is related to elective procedures such as panniculectomy or others procedures that were previously deferred due to patient's obesity.

\section{Limitation of Study}

This was a retrospective study that was limited by its small sample size, selection and information bias.

It main advantage was in attempting to address the all-important question of safety of RYGB in the adolescent population.

\section{Conclusion}

Laparoscopic RYGB in adolescent population was associated with excess weight loss of $74 \%$ at 2 years, a robust disease resolution (75-100\%) and a low complication profile. Although our sample size was small, we believe that it is a safe procedure and offers a suitable alternative in morbidly obese adolescents.

\section{References}

1. Rosenbaum M (2007) Epidemiology of pediatric obesity. Pediatr Ann 36: 8995.

2. Whitaker RC, Wright JA, Pepe MS, Seidel KD, Dietz WH (1997) Predicting obesity in young adulthood from childhood and parental obesity. $N$ Engl J Med 337: 869-873.

3. Guo SS, Huang C, Maynard LM, Demerath E, Towne B, et al. (2000) Body mass index during childhood, adolescence and young adulthood in relation to adult overweight and adiposity: the Fels Longitudinal Study. Int J Obes Relat Metab Disord 24: 1628-1635.

4. Serdula MK, Ivery D, Coates RJ, Freedman DS, Williamson DF, et al. (1993) Do obese children become obese adults? A review of the literature. Prev Med 22: 167-177.

5. Puzziferri N, Nakonezny PA, Livingston EH, Carmody TJ, Provost DA, et al. (2008) Variations of weight loss following gastric bypass and gastric band. Ann Surg 248: 233-242

6. O'Brien PE, Sawyer SM, Laurie C, Brown WA, Skinner S, et al. (2010) Laparoscopic adjustable gastric banding in severely obese adolescents: a randomized trial. JAMA 303: 516-526.

7. Chapman AE, Kiroff G, Game P, Foster B, O'Brien P, et al. (2004) Laparoscopic adjustable gastric banding in the treatment of obesity: a systematic literature review. Surgery 135: 326-351.

8. Zehetner J, Holzinger F, Triaca H, Klaiber Ch (2005) A 6-year experience with the Swedish adjustable gastric band Prospective long-term audit of laparoscopic gastric banding. Surg Endosc 19: 21-28.

9. Inge TH, Krebs NF, Garcia VF, Skelton JA, Guice KS, et al. (2004) Bariatric surgery for severely overweight adolescents: concerns and recommendations. Pediatrics 114: 217-223.

10. August GP, Caprio S, Fennoy I, Freemark M, Kaufman FR, et al. (2008) Prevention and treatment of pediatric obesity: an endocrine society clinical practice guideline based on expert opinion. J Clin Endocrinol Metab 93: 45764599.

11. NHANES 1999-2000 public data release file documentation, p: 1-6.

12. Centers for Disease Control and Prevention (2015) NHANES 1999-2000.

13. NHANES 1999-2000 Addendum to the NHANES III analytical guidelines, p: 1-28. 
Citation: Agaba EA, Rainsville H, Vemulapalli P. Pediatric Gastric Bypass: Is it Time to Rethink? Lessons Learned from a Single Institution Experience.. J Obes Bariatrics. 2015;2(1): 5.

ISSN: $2377-9284$

14. Jen HC, Rickard DG, Shew SB, Maggard MA, Slusser WM, et al. (2010) Trends and outcomes of adolescent bariatric surgery in California, 20052007. Pediatrics 126: e746-753.

15. Stanford A, Glascock JM, Eid GM, Kane T, Ford HR, et al. (2003) Laparoscopic Roux-en-Y gastric bypass in morbidly obese adolescents. J Pediatr Surg 38: 430-433

16. Suter M, Calmes JM, Paroz A, Giusti V (2006) A 10- year experience with laparoscopic gastric banding for morbid obesity: high long-term complication and failure rates. Obes Surg 16: 829-835.

17. Lyass S, Cunneen SA, Hagiike M, Misra M, Burch M, et al. (2005) Devicerelated reoperations after laparoscopic adjustable gastric banding. Am Surg 71: 738-743.

18. Anderson AE, Soper RT, Scott DH (1980) Gastric bypass for morbid obesity in children and adolescents. J Pediatr Surg 15: 876-881.

19. Rand CS, MacGregor AM (1994) Adolescents having obesity surgery: a 6-year follow-up. South Med J 87: 1208-1213.

20. Higa KD, Boonne KB, Ho T (2000) Complications of the laparoscopic Rouxen-Y gastric bypass: 1040 patients- what have we learned? Obes Surg 10: 509-513.

21. Schauer PR, Ikramuddin S (2001) Laparoscopic surgery for morbid obesity Surg Clin North Am 81: 1145-1179.

22. Podnos YD, Jimenez JC, Wilson SE, Stevens CM, Nguyen NT (2003) Complications after laparoscopic gastric bypass: a review of 3464 cases. Arch Surg 138: 957-961.

23. Agaba EA, Shamseddeen H, Gentles CV, Sasthakonar V, Gellman L, et al (2008) Laparoscopic vs open gastric bypass in the management of morbid obesity: a 7-year retrospective study of 1,364 patients from a single center. Obes Surg 18: 1359-1363.

24. Eid GM, Cottam DR, Velcu LM, Mattar SG, Korytkowski MT, et al. (2005) Effective treatment of polycystic ovarian syndrome with Roux-en-Y gastric bypass. Surg Obes Relat Dis 1: 77-80.

25. Wittgrove AC, Clark GW (2000) Laparoscopic gastric bypass, Roux-en-Y: 500 patients: technique and results, with 3-60 month follow-up. Obes Surg 10: 233-239.

26. Nguyen NT, Goldman C, Rosenquist CJ, Arango A, Cole CJ, et al. (2001) Laparoscopic versus open gastric bypass: a randomized study of outcomes, quality of life, and costs. Ann Surg 234: 279-289.

27. Higa KD, Ho T, Boone KB (2001) Laparoscopic Roux-en-Y gastric bypass: technique and 3-year follow-up. J Laparoendosc Adv Surg Tech A 11: 377 382.

28. Tsai WS, Inge TH, Burd RS (2007) Bariatric surgery in adolescents: recent national trends in use and in-hospital outcome. Arch Pediatr Adolesc Med 161: $217-221$.

29. Service GJ, Thompson GB, Service FJ, Andrews JC, Collazo-Clavell ML, et al. (2005) Hyperinsulinemic hypoglycemia with nesidioblastosis after gastricbypass surgery. N Engl J Med 353: 249-254.

30. Patti ME, McMahon G, Mun EC, Bitton A, Holst JJ, et al. (2005) Severe hypoglycemia post-gastric bypass requiring partial pancreatectomy: evidence for inappropriate insulin secretion and pancreatic islet hyperplasia. Diabetologia 48: 2236-2240.

31. Zingmond DS, McGory ML, Ko CY (2005) Hospitalization before and after gastric bypass surgery. JAMA 294: 1918-1924. 